\title{
Biomimetic water-collecting fabric with light-induced superhydrophilic bumps
}

Yuanfeng Wang, Xiaowen Wang, Chuilin Lai, Huawen Hu, Yeeyee Kong,

Bin Fei and John H. Xin*

Nanotechnology Centre, Institute of Textiles and Clothing, The Hong Kong

Polytechnic University, Hong Kong SAR 999077, China.

Corresponding Author

*Fax: +86-852-2766-6474. E-mail: tcxinjh@polyu.edu.hk. 


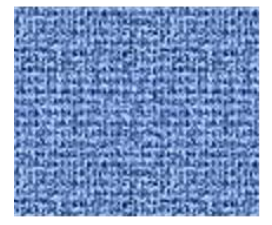

Bare-C

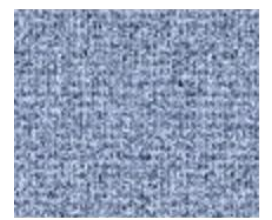

TC

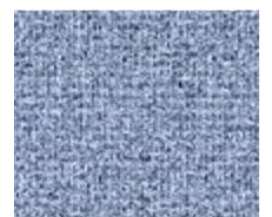

TC-UV

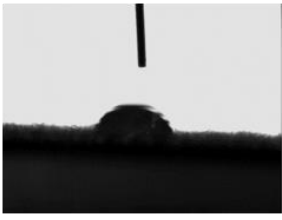

$0.15 \mathrm{~s}$

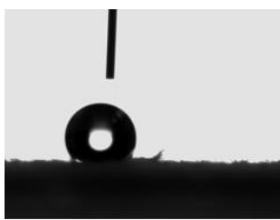

$0.16 \mathrm{~s}$

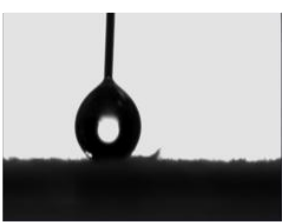

$0 \mathrm{~s}$

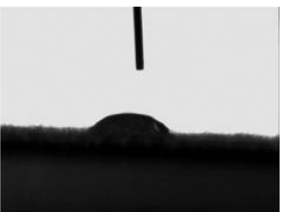

$0.20 \mathrm{~s}$

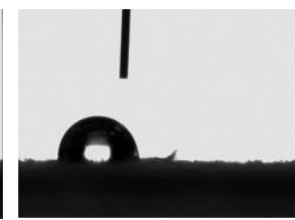

$1.50 \mathrm{~s}$

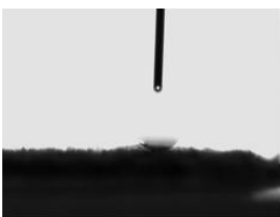

$0.05 \mathrm{~s}$

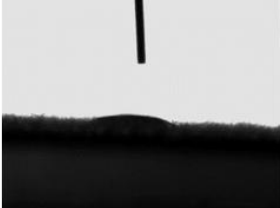

$0.31 \mathrm{~s}$

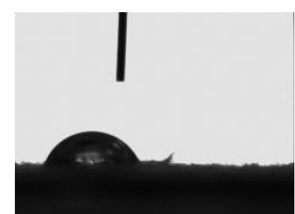

$2.28 \mathrm{~s}$

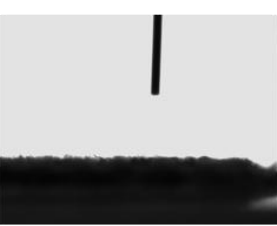

$0.16 \mathrm{~s}$

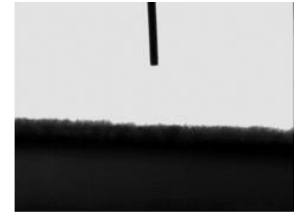

$0.56 \mathrm{~s}$

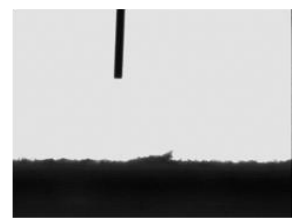

$6.00 \mathrm{~s}$

Figure S1. Illustration of the hydrophilicity of bare-C, TC, and UV-treated TC (TC-UV). Time needed for water droplets to become $0^{\circ}$ on bare-C, TC and TC-UV is $0.56 \mathrm{~s}, 6 \mathrm{~s}$ and $0.16 \mathrm{~s}$ respectively. A sunlight irradiation of $1 \mathrm{~h}$ was confirmed to have the same result (with a light intensity being $1 \mathrm{~mW} / \mathrm{cm}^{2}$ ). 


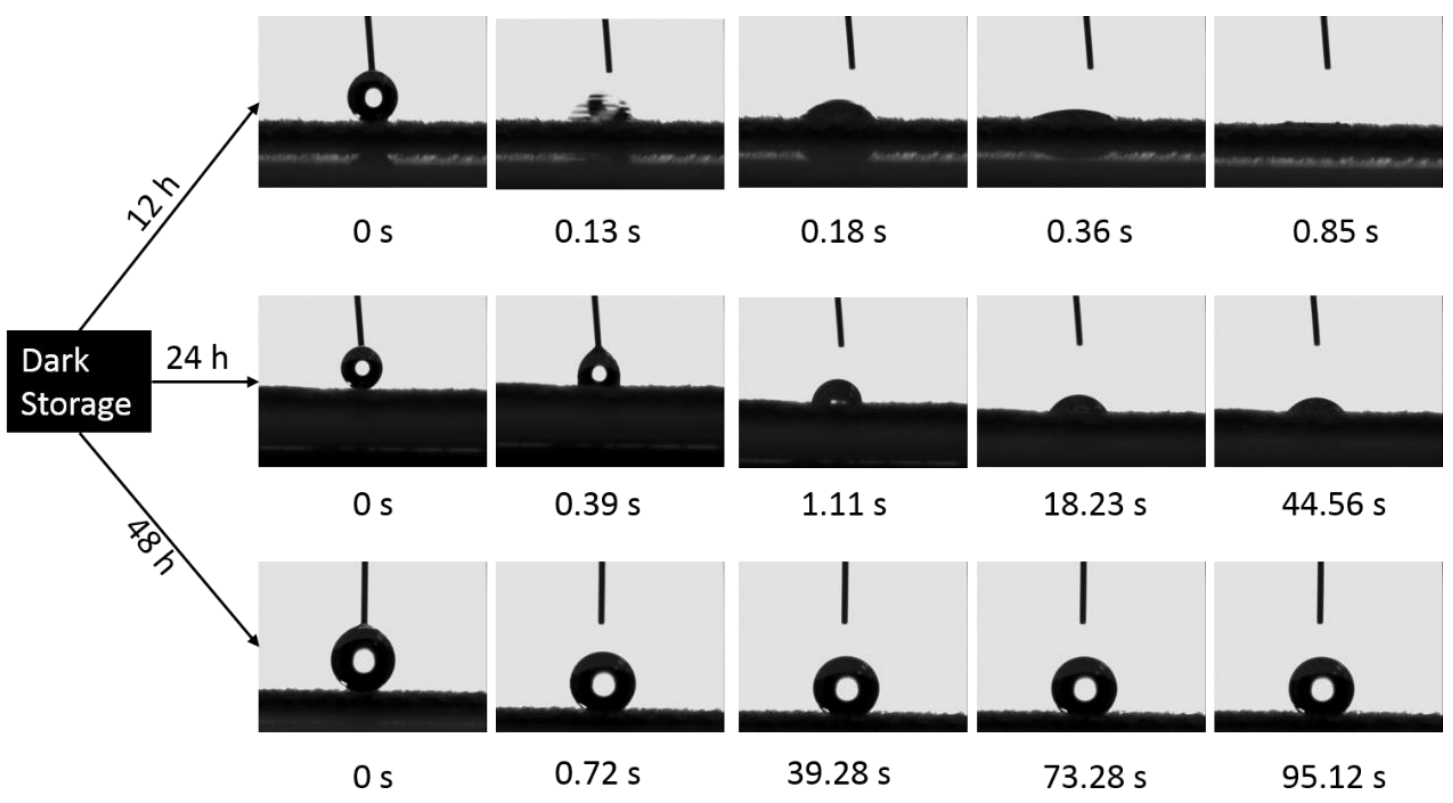

Figure S2. Pictures of water droplets on TC fabric after UV irradiation and following dark-storage for $12 \mathrm{~h}, 24 \mathrm{~h}$ and $48 \mathrm{~h}$. After $12 \mathrm{~h}$ dark-storage, $\mathrm{TiO}_{2}$ maintains superhydrophilic and only $0.85 \mathrm{~s}$ is needed for water droplets to infilter into the TC fabric. The hydrophilicity of TC fabric decreases apparently after more than $24 \mathrm{~h}$ dark-storage, and even turn hydrophobic with a WCA around $144.6^{\circ}$ after $48 \mathrm{~h}$ of dark-storage. 

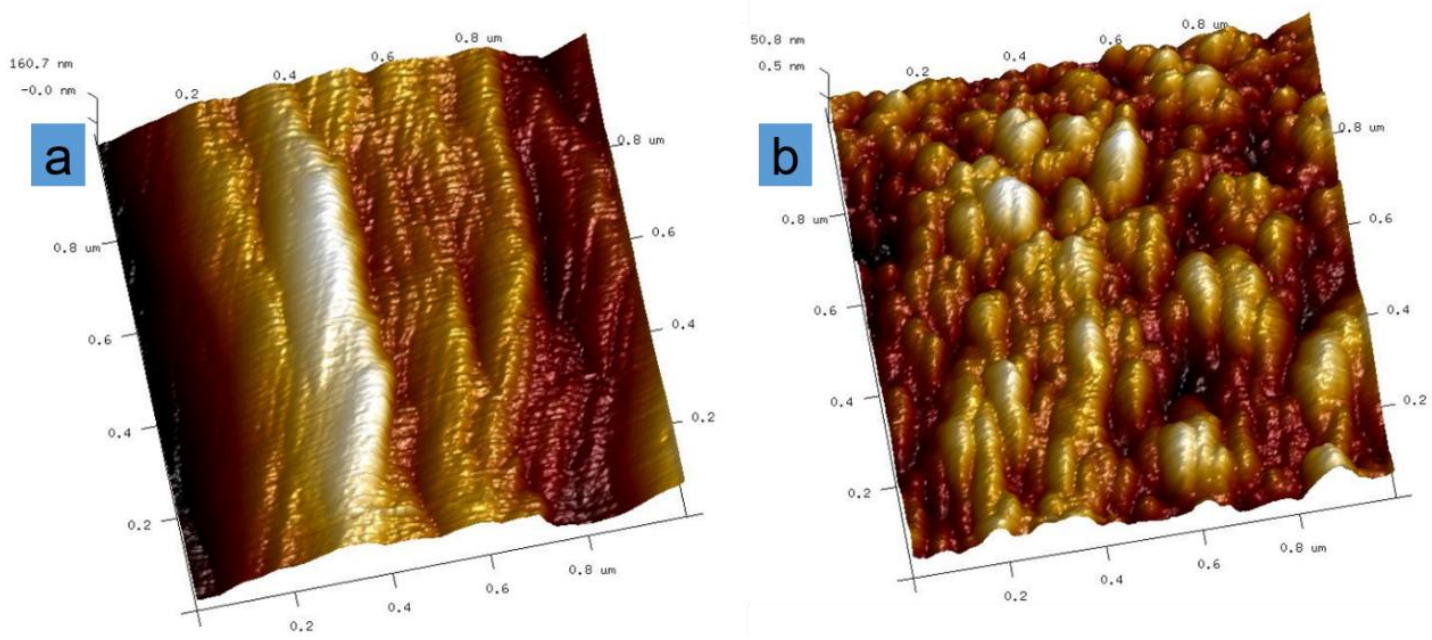

Figure S3. AFM images of bare-C (a) and SC (b) fabrics in tapping mode.
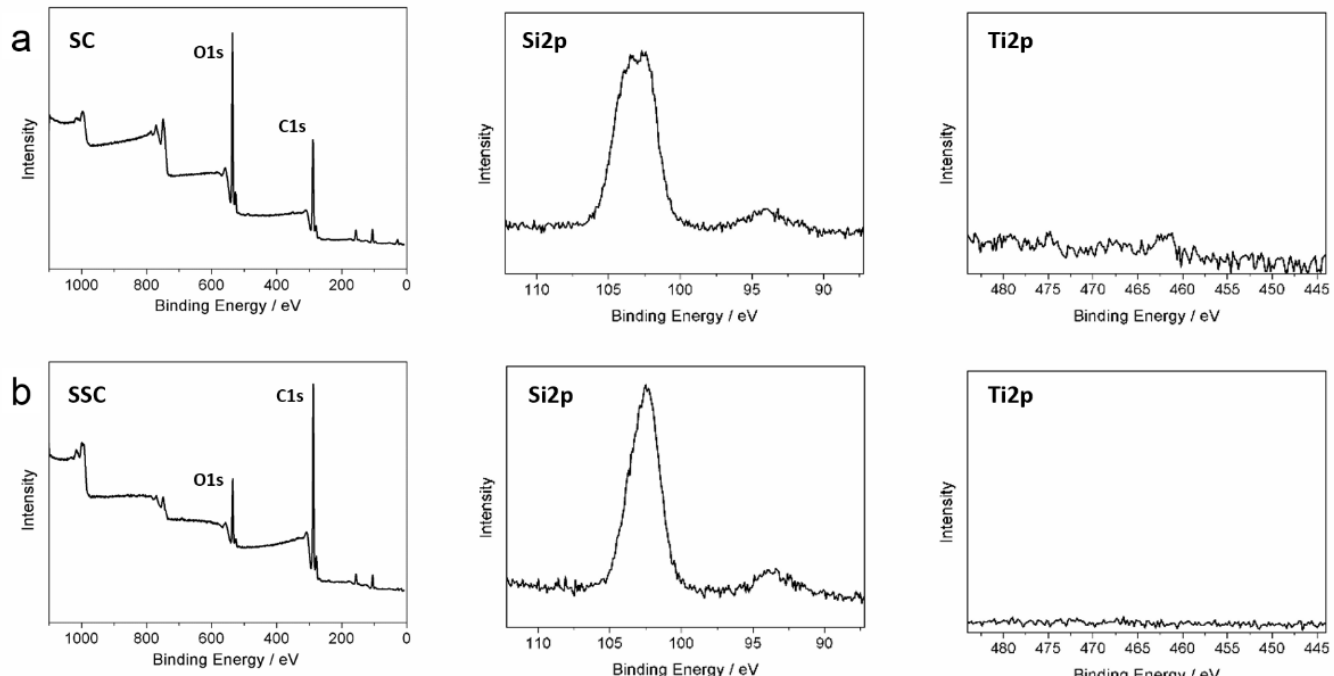

Figure S4. XPS results of the prepared SC (a) and SSC (b) fabrics. The far left column are integral spectra of the fabrics and the others are spectra with high-resolution denoting $\mathrm{Si}$ and Ti elements as labeled in the spectra. 

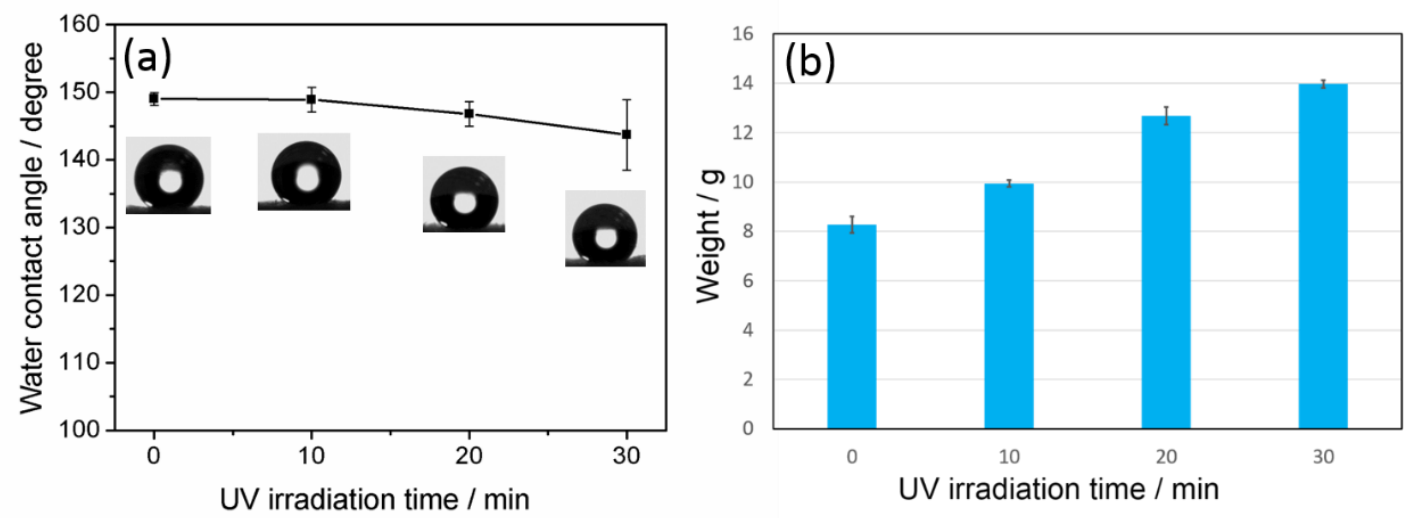

Figure S5. (a) Gradual WCA decrease on SSTC-11 fabric surface along with an increasing time of UV irradiation; (b) Water collection performance (for $1 \mathrm{~h}$ ) of SSTC-11 fabric after different time of UV irradiation.

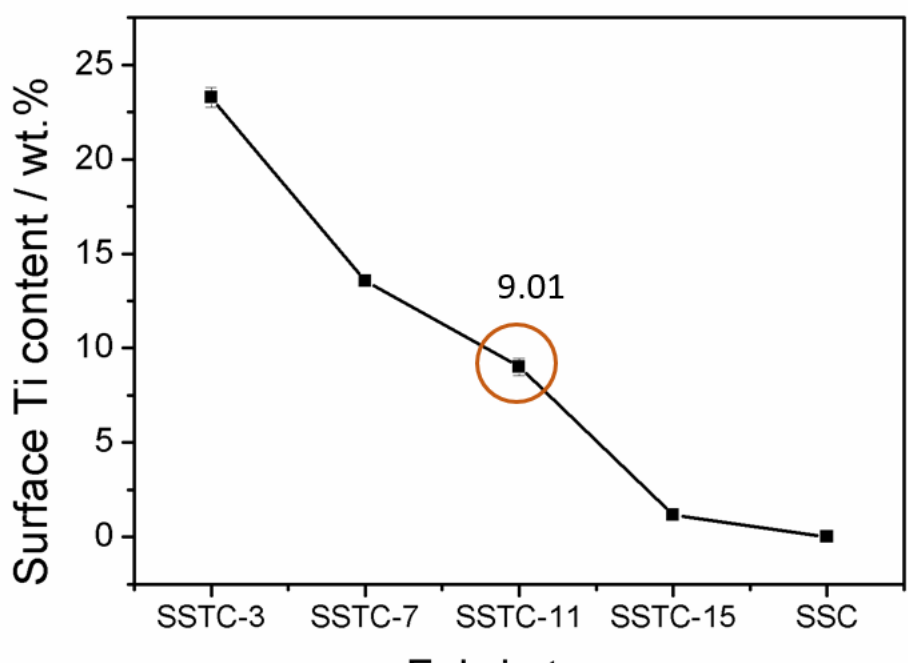

Fabric type

Figure S6. Surface Ti content of SSTC-3, SSTC-7, SSTC-11, SSTC-15, and SSC fabric detected by EDX. 

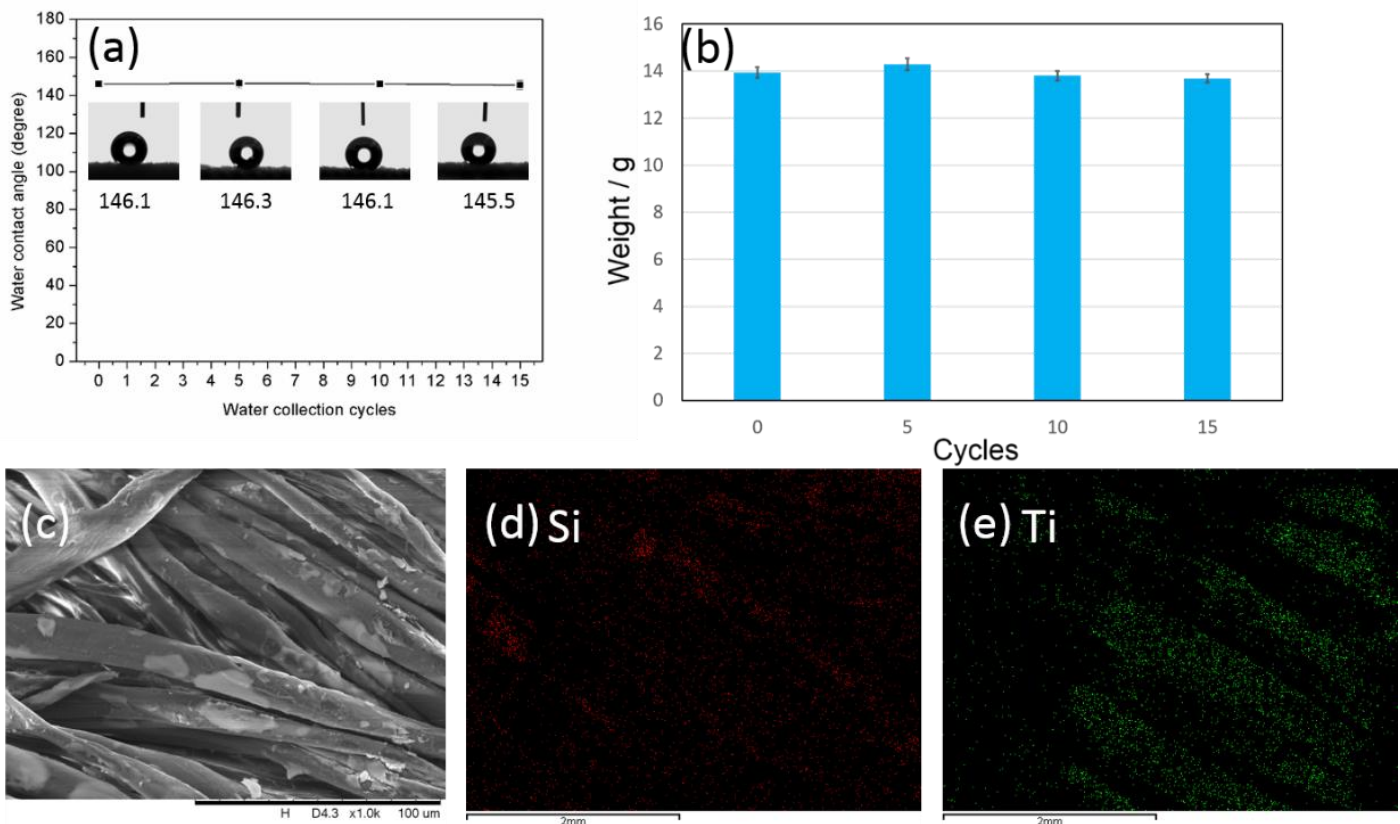

Figure S7. (a) Line graph displaying the relationship between WCA and water collection cycles (insets show the WCAs after $0,5,10$, and 15 cycles of water collection); (b) Water collection performance/weight after $0,5,10$, and 15 cycles of water-collecting repetition; (c) SEM image of the SSTC fabric surface after 15 cycles of water collection; (d) and (e) are EDS mappings of Si and Ti, respectively, on SSTC fabric after 15 cycles of water collection. 

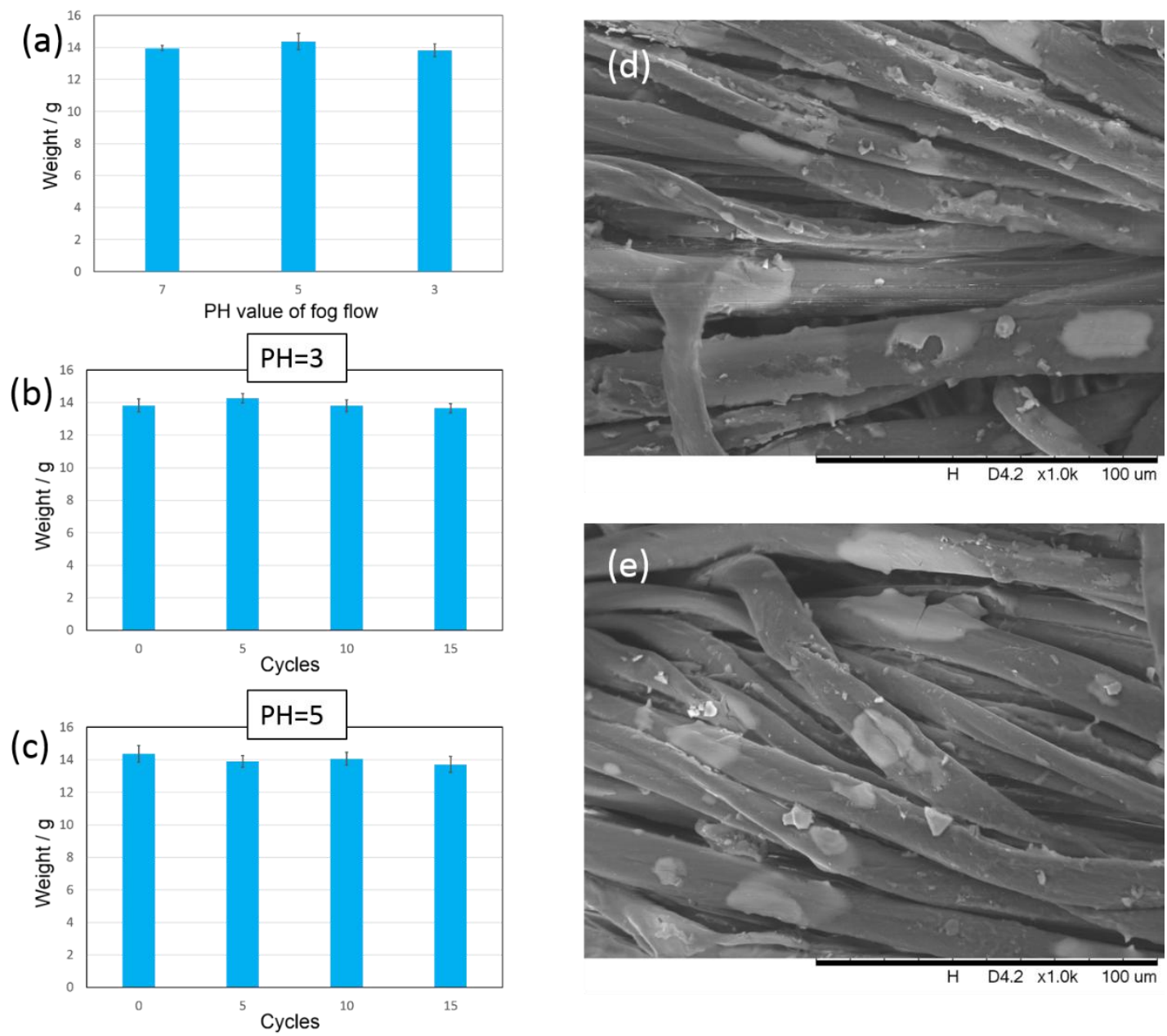

Figure S8. (a) Water collection stability of the as-prepared SSTC-11 fabric under acidic fog flow with $\mathrm{pH}$ values of 3 and 5 (with DI water generated fog as reference); (b) Water collection stability after $0,5,10$, and 15 cycles under fog flow with $\mathrm{pH}$ values of 3 ; (c) Water collection stability after $0,5,10$, and 15 cycles under fog flow with pH values of 5; (d) SEM image of the SSTC-11 fabric surface after 15 cycles of water collection under fog flow with $\mathrm{pH}$ values of 3; (e) SEM image of the SSTC-11 fabric surface after 15 cycles of water collection under fog flow with $\mathrm{pH}$ values of 5 . 

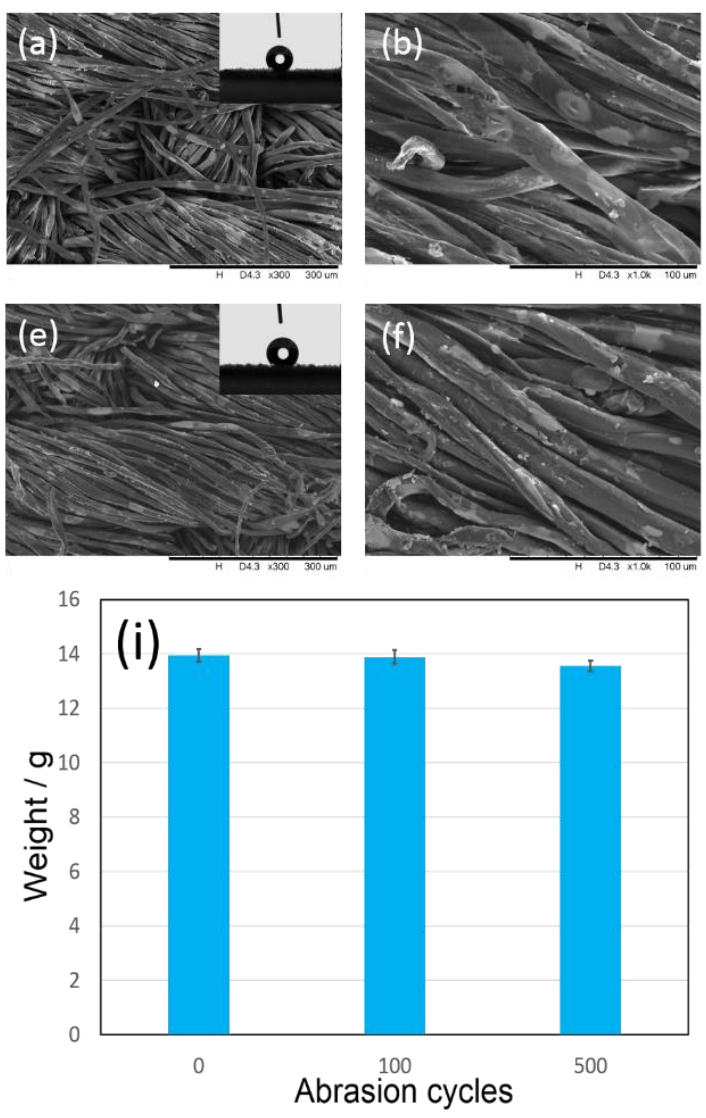
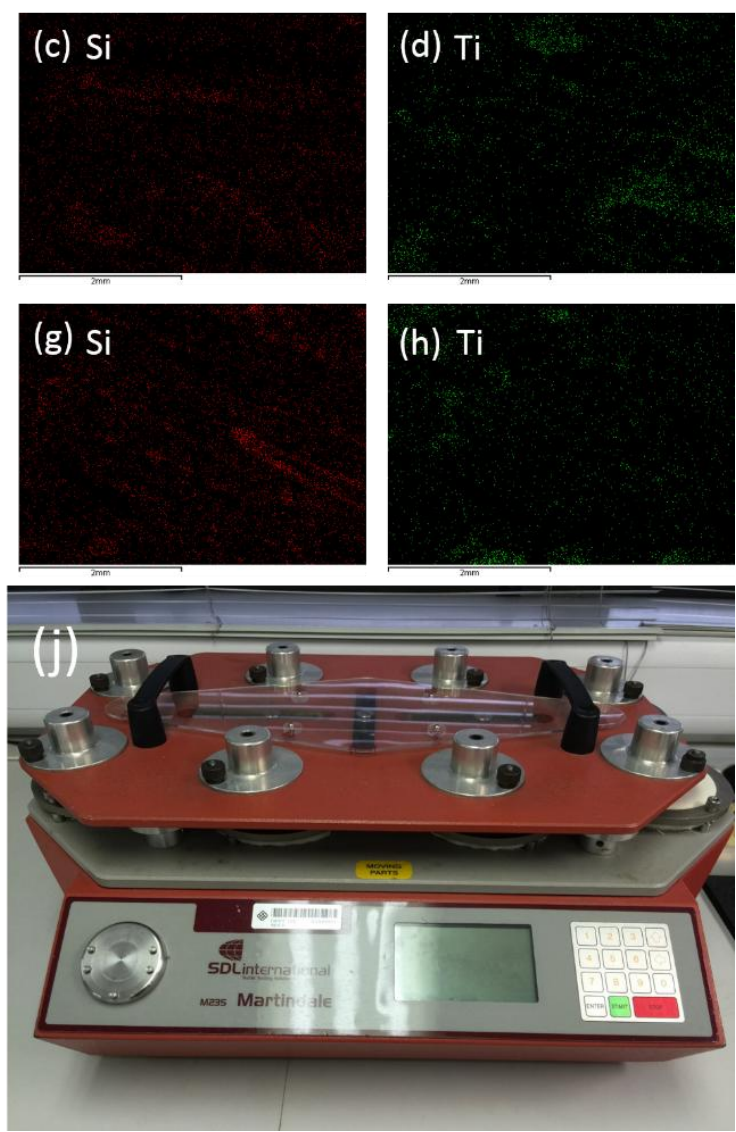

Figure S9. Rub resistance testing results of the superhydrophobic coating and the $\mathrm{TiO}_{2}$ bumps on the SSTC-11 fabric. (a)-(d) are SEM images with magnification of x300, x1000, EDS mapping of Si, and Ti on SSTC fabric surface after 100 times of friction respectively; (e)-(h) are SEM images with magnification of $\mathrm{x} 300, \mathrm{x} 1000$, EDS mapping of Si, and Ti on SSTC fabric surface after 500 times of friction respectively, insets in (a) and (e) show the after-UV WCAs of SSTC after 100 and 500 times friction respectively; (i) shows the water collection performance/weight after 0,100 , and 500 times abrasion; (j) is the picture of Martindale abrasion-testing machine used in this article. 


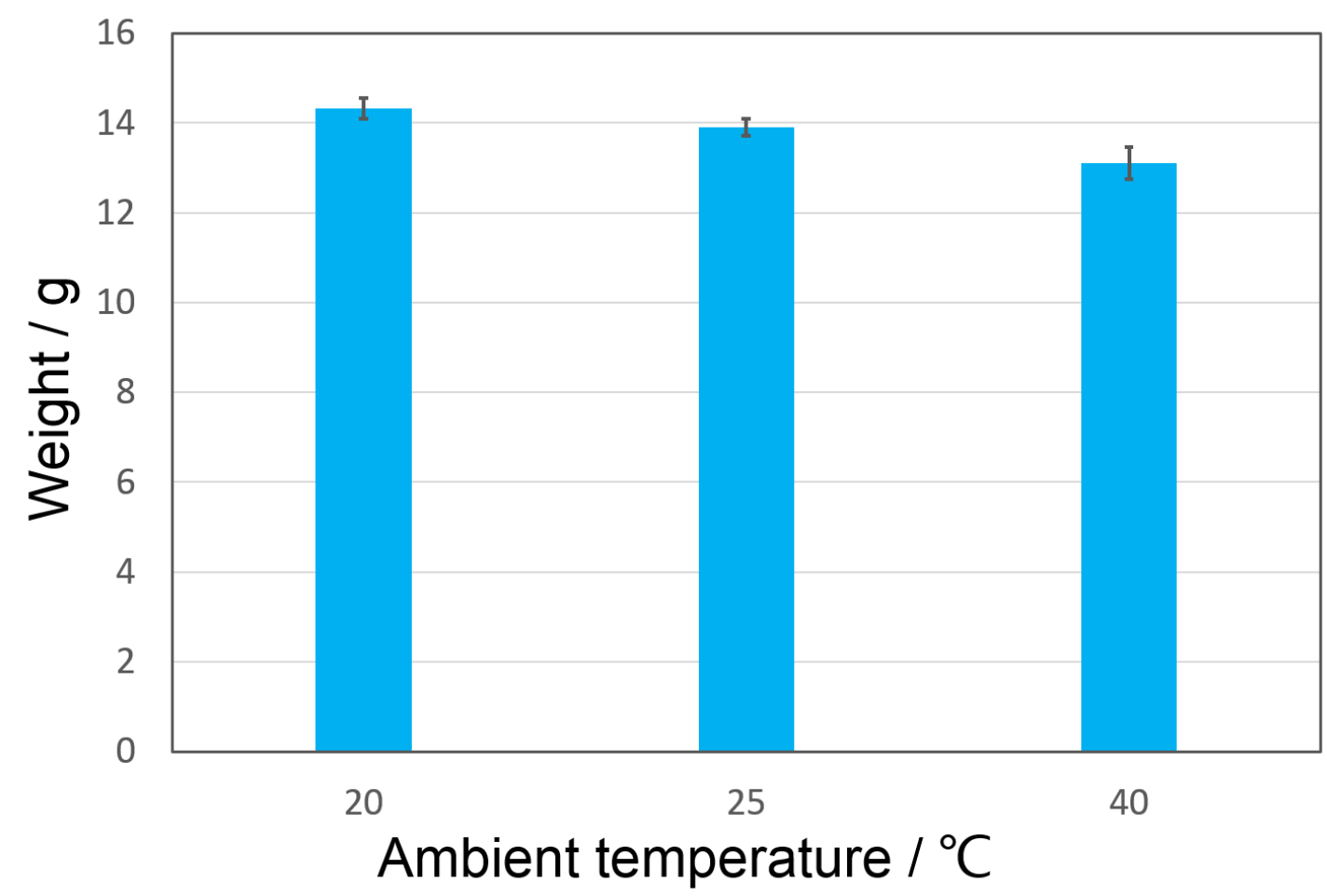

Figure S10. Water collection performance at different ambient temperature. 